\title{
Designing of sliding mode observer for the position control for quadrotor UAV
}

\author{
DING LI \\ Xi'an Aeronautical University Xi'an, Shaanxi China. \\ liding900712@126.com
}

\begin{abstract}
Aiming at the model uncertainty in system and vulnerability to external environment disturbance for the quadrotor UAV in field flight, this article uses the following ideas to solve them. Firstly, use the Newton-Euler method to establish the system model. Then, according to the inner and outer loop control structure, use the attitude command of the output of outer loop position controller as the input of inner loop attitude controller. This paper concentrates to designing of sliding mode observer controller. In addition, the stability of position subsystem proved by Lyapunov theory, the resulted conclusion state that the error converges to zero in finite time. Finally, the simulation of fixed-point control shows that the controller has fast response speed, anti-interference performance, and can track quickly and accurately.
\end{abstract}

Keywords: quadrotor, UAV controlling, SMC.

\section{Introduction}

In the recent years, unmanned aerial vehicles (UAV's) are widely used. The UAV falls into different sizes and different classes [1]. The UAV's are used for civilian purpose e.g. (atmospheric observation \& insecticide spraying) and for military purpose e.g. (communication \& targeting the point) as well. The UAV falls into two major classes (i) Multi-Rotors (ii) Fixed-Wing. The quadrotor is also called multi-rotor helicopter that is propelled by the four rotors [2]. Quadrotor/ quadcopters are classified as rotorcraft. The quadrotor has various advantages due to its simple structure and low weight. The main characteristics of quadrotor UAV are high mobility, low cost and vertical take-off and landing [3]. Quadrotor UAV are widely used for aerial photography, transportation, patrolling and protection. Quadrotor can apprehend fast and precise tracking control of under actuated system because it is multi-variable, non-linear and highly coupled [4] and [5]. Presently, the quadrotor UAV is a hot topic from the research point of view. The concept has transformed from linear control to non-linear control. The typical linear control system such as a proportional, integral, derivative (PID) control has many advantages such as easy to implement, modest controller design etc. The disadvantage is only when an external disturbance is added [6]. The technique for using non-linear control systems e.g. sliding mode control, adaptive control, backstepping control is to overcome the 
deficiencies of the linear control system [7-10]. When the deficiencies are overcome, the system has good robust performance and dynamic tracking performance. The main advantage of using non-linear control method is that it allows the model to be inaccurate [11]. One thing kept in mind while designing the control signal is that the system state reserved in sliding mode control (SMC), with the help of selected SMC, the dynamic performance of the system govern. The response of the system is insensitive to external disturbances and uncertainty when the system is in the sliding mode. Easily understand the referred state of quad-rotor UAV, the adaptive sliding mode (ASM) algorithm implemented. The virtual control law designed with the help of ASM in [12]. The main characteristic of linear sliding mode function is that the union of the system state is asymptotic at the equilibrium position [13]. The switching control idea is used to pact with the convergence only for the singularity problem. Still, there is no assurance that state error harshly touches the zero point. By considering the abovementioned problems that are uncertainty and external disturbance, a non-linear model of quadcopter/quadrotor UAV designed. The model consists of inner and outer loop control structure. The inner loop consists of cascade controller and the outer/external loop contain sliding mode observer position controller for four kinds of control. In this article, the position control of the rotor are deliberated. The main advantage of using sliding mode control observer (SMCO) is that is limits the system state error. Similarly, it has the advantage that it removes the singularity problem and strict converge the state error to zero. The above manuscript is divided in to five sections i.e. section II, defines the model of quad rotor UAV. In section III, demonstrates the designing of controller, which followed by section IV in which simulation results are discussed. Lastly, the whole article concluded in section V.

\section{Mathematical model}

The quad-rotor UAVs have two kinds of structures, which are responsible for the attitude measurements; there is not much difference between these two structures. The x-shaped structures under the four rotors are involved in the attitude control, in which the torque becomes large, the controllable margin increases [14]. In this paper, the mathematical model of $x$-shaped quad-rotor developed based on the kinematics and dynamics equations of the UAV. Following are the assumptions used in establishing the mathematical model of the UAV.

1. The aerial vehicle is a symmetrical rigid body.

2. The origin in the body coordinate system coincides with the geometric center of the four rotors.

3. The resistance and gravity of the body is independent on the flight attitude.

4. The upward force or the thrust generated by each motor is proportional to the square of the motor speed.

The movement of the aerial vehicle in three dimensions is a rigid body with 6-DOF, which can be divided into translational motion and rotational motion. On the basis of above assumptions, in order to facilitate the designing of the kinematic model of the aircraft two coordinate system are selected. The Earth's coordinate system and fixed body coordinate system. The position of the 
UAV in the Earth's coordinate system is defined as $\xi=(X, Y, Z)^{T}$, the attitude angles with respect to geographic coordinate system is $\Omega=(\varphi, \theta, \psi)^{T}$. According to Newton Euler method, the equations of translational motion in Earth's coordinate system are discussed (1). The upward force or the lift i.e. $F_{1}, F_{2}, F_{3}, F_{4}$ produced by the rotors is defined in figure 1 .

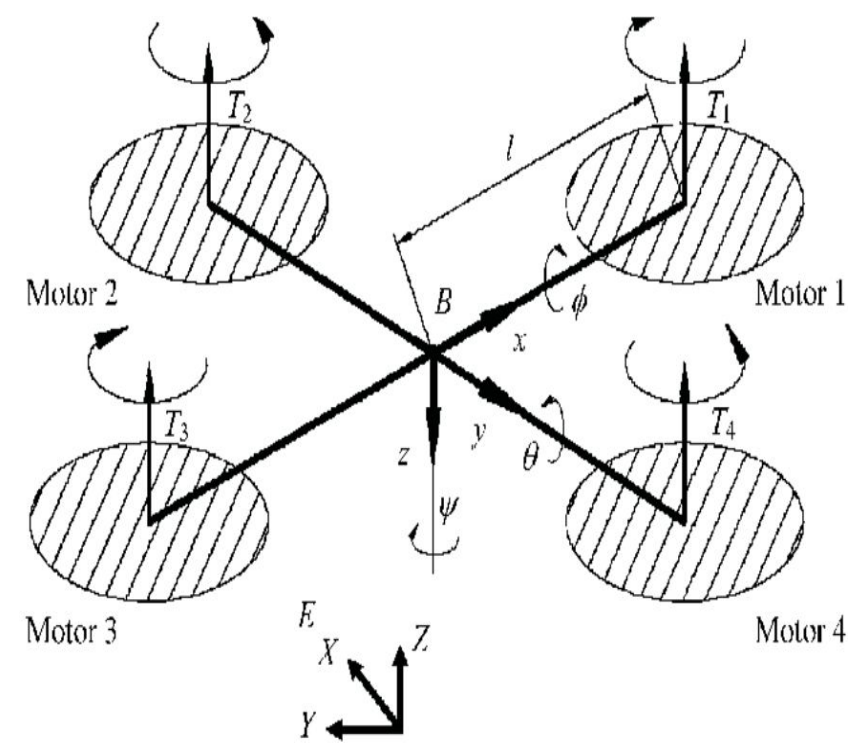

Fig. 1: Model of quad-rotor UAV [15].

The translational motion equation for the UAV is:

$$
F=F_{T}-F_{g}-F_{f}=m \ddot{\xi}
$$

Where $\mathrm{F}$ is the resultant force produced by the four rotors in space, $F_{T}$ is the lift or thrust produced by the rotors, $F_{g}=(0,0, m g)^{T}$ is the weight of four rotors and $F_{f}$ is the air resistance acting on the rotors. Where " $m$ " is the mass of the UAV. $\xi=(X, Y, Z)^{T}$ represent the translational motion of the UAV, $\ddot{\xi}$ defined for the acceleration of the four rotors. Now we can assume that the lift generated by each motor is directly proportional to the square of each motor speed. The upward lift generated by the four propellers is perpendicular to the surface of the body, the combined lift force of all the motors is converted into Earth's coordinate system by the rotation matrix.

In the geographic coordinate system, the air resistance and flight speed of the quad-rotor are directly proportional to air resistance coefficient $\left(k_{x}, k_{y}, k_{z}\right)$ of the three axis respectively. The translational equations of motion for the geographic coordinate system are: 


$$
\begin{gathered}
\ddot{X}=\left[F_{T}(\cos \varphi \sin \theta \cos \psi+\sin \varphi \sin \psi)-k_{x} X\right] / m \\
\ddot{Y}=\left[\begin{array}{c}
{\left[F_{T}(\cos \varphi \sin \theta \sin \psi+\sin \varphi \cos \psi)-k_{y} \dot{Y}\right] / m} \\
\ddot{Z}=\left[F_{T}(\cos \theta \cos \varphi)-k_{x} \dot{X}\right] / m-g
\end{array}\right.
\end{gathered}
$$

The torque of the aircraft includes rotor torque, air resistance torque, and gyroscopic torque. $I$ represent the moment of inertia. The weight and structure of a quad-rotor is assumed by the previous symmetry, so $I=\left(I_{x}, I_{y}, I_{z}\right) E$, E is the third order matrix. $I_{x}, I_{y}, I_{z}$ are three axis rotation inertia which are constants. Where $L=\left(L_{x}, L_{y}, L_{z}\right)$ is the angular momentum of the quad-rotor to its centroid, $M=\left(M_{x}, M_{y}, M_{z}\right)$ is the total external torque acting on the centroid and the angular velocity of the UAV is denoted by $\omega=\left(\omega_{x}, \omega_{y}, \omega_{z}\right)$. The rotational equations for the motion of quad-rotor according to angular momentum theorem discussed below.

$$
M=\frac{d L}{d t}=\left(\frac{d L_{x}}{d t} i+\frac{d L_{y}}{d t} j+\frac{d L_{z}}{d t} k\right)+\quad\left(L_{x} \frac{d i}{d t}, L_{y} \frac{d i}{d t}\right), L_{z} \frac{d i}{d t}
$$

The angular momentum of the aerial vehicle on its center of mass is equal to the product of the moment of inertia and the aangular velocity, therefore can be obtained:

$$
\begin{aligned}
& M_{x}=I_{x} \frac{d \omega_{x}}{d t}+\left(I_{z}-I_{y}\right) \omega_{y} \omega_{z} \\
& M_{y}=I_{y} \frac{d \omega_{y}}{d t}+\left(I_{x}-I_{z}\right) \omega_{z} \omega_{x} \\
& M_{z}=I_{z} \frac{d \omega_{z}}{d t}+\left(I_{y}-I_{x}\right) \omega_{x} \omega_{y}
\end{aligned}
$$

Now, $\left(U_{1}, U_{2}, U_{3}\right)$ defines the roll moment, yaw moment and pitch moment. Therefore, the torque generated by the rotor is:

$$
M_{1}=\left(\begin{array}{c}
U_{1} \\
U_{2} \\
U_{3}
\end{array}\right)=\left(\begin{array}{l}
F_{1}+F_{4}-F_{2}-F_{3} \\
F_{1}+F_{2}-F_{3}-F_{4} \\
F_{1}+F_{3}-F_{2}-F_{4}
\end{array}\right)=\left(\begin{array}{c}
\frac{\sqrt{2}}{2} k_{b} l\left(\omega_{1}^{2}+\omega_{4}^{2}-\omega_{2}^{2}-\omega_{3}^{2}\right) \\
\frac{\sqrt{2}}{2} k_{b} l\left(\omega_{1}^{2}+\omega_{2}^{2}-\omega_{3}^{2}-\omega_{4}^{2}\right. \\
k_{d}\left(\omega_{2}^{2}+\omega_{4}^{2}-\omega_{1}^{2}-\omega_{3}^{2}\right.
\end{array}\right)
$$

Where $l$ is the distance between the centers of propeller to the center of the UAV $M_{1}$ is the total torque and $k_{d}$ is the inverse torque factor of the motor. In the previous hypothesis, the air resistance of the airframe was not affected by the flight attitude, visual air resistance torque is proportional to the angular velocity of the posture among them $k_{\varphi}, k_{\theta}, k_{\psi}$ were four rotor drag torque coefficient. In this article, the attitude angle of the aircraft and the rotation rate is very small; therefore, it can be consider that the rotation angular velocity is equal to attitude angular velocity on the three axes of the body coordinate system. The rotational motion equations for the flight-controller of UAV are as follows [16]; 


$$
\begin{aligned}
& \ddot{\varphi}=\left[\left(I_{x}-I_{z}\right) \dot{\theta} \dot{\psi}+I_{b} \dot{\theta} \omega_{r}+U_{2}-k_{\varphi} \dot{\varphi}\right] / I_{x} \\
& \ddot{\theta}=\left[\left(I_{z}-I_{x}\right) \dot{\varphi} \dot{\psi}-I_{b} \dot{\varphi} \omega_{r}+U_{3}-k_{\theta} \dot{\theta}\right] / I_{y} \\
& \ddot{\psi}=\left[\left(I_{x}-y\right) \dot{\theta} \dot{\varphi}+I_{b} \dot{\theta} \omega_{r}+U_{4}-k_{\psi} \dot{\psi}\right] / I_{z}
\end{aligned}
$$

The model will simplify now by ignoring the influence of air gravity and helical torque. The fixed body coordinates spindle rotational inertia difference can be obtained by UAV's power equations [17];

$$
\begin{gathered}
\ddot{X}=(\cos \varphi \sin \theta \cos \psi+\sin \varphi \sin \psi) U_{1} / m \\
\ddot{Y}=(\cos \varphi \sin \theta \sin \psi-\sin \varphi \cos \psi) U_{1} / m \\
\ddot{Z}=(\cos \theta \cos \varphi) U_{1} / m-g \\
\ddot{\varphi}=U_{1} / I_{x} \\
\ddot{\theta}=U_{2} / I_{y} \\
\ddot{\psi}=U_{3} / I_{z}
\end{gathered}
$$

\section{Designing of controller}

In this research article, as discussed earlier the outer and inner loop used to design the quadcopter control system. Figure 2 shows the control block diagram of the system.

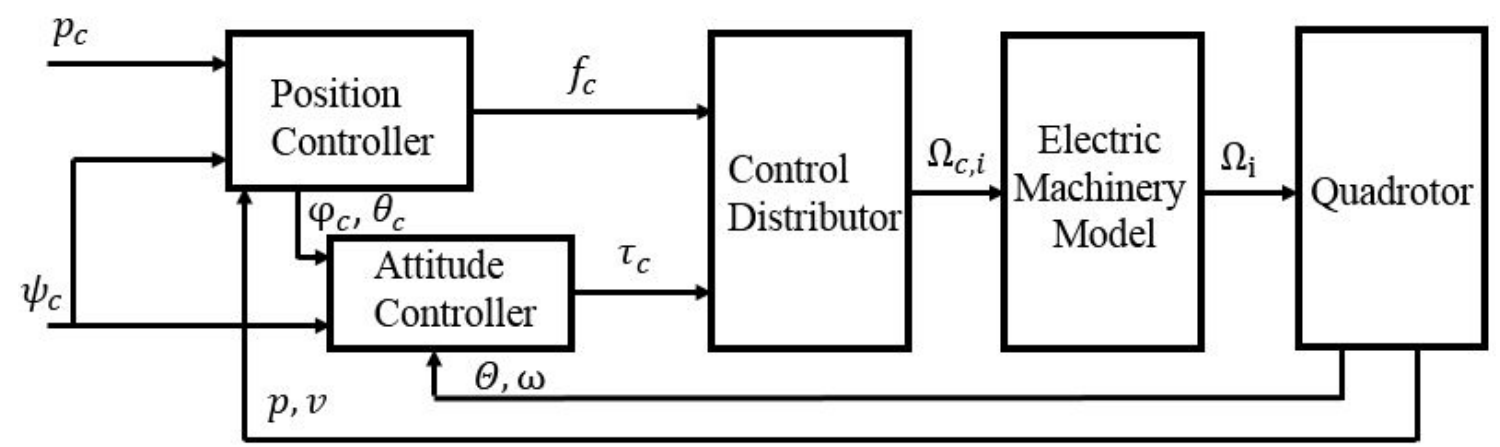

Fig. 2: Quad Control Block Diagram

In figure 2, the subscript shows the expected/anticipation value. Rotor model of UAV can simplify the transfer function (T.F) of the filter about $1.05 \% \Omega_{i}=\Omega_{c, i} / T_{m} s+1$ where $T_{m}$ is the response time constant of motor. During the flight of UAV, consider the small change of attitude angle at the point $\dot{\Theta}=\omega$. The yaw angle $\psi_{c}$ and the position $p_{c}$ obtained by the 
reference trajectory. The attitude controller gives the attitude value $\varphi_{c}$. The position controller generates the altitude force $f_{c}$ and the angle $\theta_{c}$. The position taken by the attitude controller, which controlled by these equations;

$$
\left.\begin{array}{rl}
U_{x} & =-\frac{f}{m}(\cos \psi \sin \theta \cos \varphi+\sin \psi \sin \varphi) \\
U_{y} & =-\frac{f}{m}(\sin \psi \sin \theta \cos \varphi-\cos \psi \sin \varphi) \\
U_{z} & =g-\frac{f}{m}(\cos \theta \cos \varphi)
\end{array}\right\}
$$

Formerely, the altitude and attitude command can be calculated as:

$$
\left.\begin{array}{r}
f_{c}=m \sqrt{U_{x^{2}}+U_{y^{2}}+\left(g-U_{z}\right)^{2}} \\
\varphi_{c}=\arcsin \left(-\frac{m}{f_{c}}\left(U_{x} \sin \psi_{c}-U_{y} \cos \psi_{c}\right)\right) \\
\theta_{c}=\arctan \left(\frac{U_{x} \cos \psi_{c}+U_{y} \sin \psi_{c}}{U_{z}-g}\right)
\end{array}\right\}
$$

Consider that the simpilifictaion of the model, change or airflow and anaccuate parameters of the system would thirsty the system. The translation mathematical model of quadrotor UAV can be given as:

$$
\left.\begin{array}{l}
\ddot{x}=U_{x}+\Delta f_{x}+d_{x} \\
\ddot{y}=U_{y}+\Delta f_{y}+d_{y} \\
\ddot{z}=U_{z}+\Delta f_{z}+d_{z}
\end{array}\right\}
$$

\section{Design of sliding mode observer}

The second order general form of the position and attitude fault objects can be written as:

$$
\left.\begin{array}{c}
\dot{y}_{1}=y_{2} \\
\dot{y}_{2}=f(y)+g(y) u+f_{a}+\alpha
\end{array}\right\}
$$

In equation (11), $\left[y_{1}, y_{2}\right]^{T}$ repreents the systems state vectors, the continous diffrentiable functions are $f(y)$ and $g(y), f_{a}$ is the bounded fault and $\alpha$ is the constant. The sliding mode observer can be designed as

$$
\begin{gathered}
\left.\begin{array}{c}
\dot{\hat{y}}_{1}=\hat{y}_{2}+V_{1} \\
\dot{\hat{y}}_{2}=f(y)+\begin{array}{c}
g(y) u+f_{a}+\hat{\alpha}+V_{2} \\
\hat{\hat{\alpha}}=V_{3}
\end{array}
\end{array}\right\} \\
V_{1}=\beta_{1} \operatorname{sign}\left(e_{1}\right), V_{2}=\beta_{2} \operatorname{sign}\left(V_{1}\right), V_{3}=\beta_{3} \operatorname{sign}\left(e_{3}\right)
\end{gathered}
$$


In the above-mentioned equation (12) and (13), $V_{1}, V_{2}$ and $V_{3}$ are the stuffs to be designed; $\beta_{1}, \beta_{2}$ and $\beta_{3}$ are the parameters to be designed; the actual value of state $y_{1}$ is $e_{1}=y_{1}-\hat{y}_{1}$ which represents the error among the measured value $\dot{y}_{1} ; e_{3}=\alpha_{1}-\hat{\alpha}_{1}$ signify the error among the actual disturbance and observed value. The sliding mode observer consist of three states $\hat{y}_{1}$, $\hat{y}_{2}$ and $\hat{\alpha}$. The symbol function sign is substituted by its smooth form $\operatorname{sign}(Y)=Y /(|Y|+\Delta)$ to avoid the chattering taking $\Delta=0.001$.

Proof:

The error system attain by subtracting equation (11) from (12)

$$
\dot{e}_{1}=e_{2}-V_{1}, \dot{e}_{2}=f_{a}+e_{3}-V_{2}, \dot{e}_{3}=\alpha-V_{3}
$$

In the equation (14), $e_{2}=y_{2}-\hat{y}_{2}$ represent the error among the actual value and observed value and verifies $\left|e_{2}\right| \leq \gamma_{1} \hat{y}_{2} \cdot \gamma_{1}$ is the normal number.

\section{Step 1}

Define $\tilde{\gamma}_{1}=\gamma_{1}-\hat{\gamma}_{2}$ as the estimation error of the upper bound $e_{2}$, then there is $\dot{\tilde{\gamma}}=-\dot{\hat{\gamma}}$. Select the Lyapunov candidate function i.e.

$$
v_{1}=\frac{1}{2} e_{1}^{2}+\frac{1}{2 \delta_{1}} \gamma_{1}^{-2}
$$

In the abovemrntioned equation, $\delta_{1}$ is the normal number. Deriving equation (15) and subsituting equations (13) and (14) we get

$$
\dot{v}_{1} \leq\left(\gamma_{1}-\beta_{1}\right)\left|e_{1}\right|-\frac{1}{\delta_{1}} \tilde{\gamma}_{1} \dot{\hat{\gamma}}_{1}
$$

Assume $\beta_{1}=\hat{\gamma}_{1}+\varepsilon_{1}, \dot{\hat{\gamma}}_{1}=\delta_{1}\left|e_{1}\right|, \varepsilon_{1}$ is the normal number. By subsituting equation (16), we attain $\dot{v}_{1}=-\varepsilon_{1}\left|e_{1}\right| \leq 0$ representing that $e_{1} \&$ its derivative are in step 1 which reaches the 0 point in the design. Therefore, $e_{2} \rightarrow V_{1}$, formerly $V_{2}=\beta_{2} \operatorname{sign}\left(e_{2}\right)$.

\section{Step 2}

Consider the Lyapunov candidate function as $v_{15}=\frac{1}{2} e_{2}^{2}$. The derivative is attained by $\dot{v}_{15}=e_{2}\left(f_{a}+e_{3}-V_{2}\right)$ where $\left|f_{a}+e_{3}\right| \leq \gamma_{2}$. Choosing the Lyapunov candidate function

$$
v_{2}=v_{15}+\frac{1}{2 \delta_{2}} \tilde{\gamma}_{2}^{2}
$$

Deriving equation (17) and substituting equations (13) and (14) we get

$$
\dot{v}_{2} \leq\left(\gamma_{2}-\beta_{2}\right)\left|e_{2}\right|-\frac{1}{\delta_{2}} \tilde{\gamma}_{2} \dot{\hat{\gamma}}_{2}
$$


Assume $\beta_{2}=\hat{\gamma}_{2}+\varepsilon_{2}, \dot{\hat{\gamma}}_{2}=\delta_{2}\left|e_{2}\right|, \varepsilon_{2}$ is the normal number. By subsituting equation (18), we attain $\dot{v}_{2}=-\varepsilon_{2}\left|e_{2}\right| \leq 0$ representing that $e_{2} \&$ its derivative are in step 2 which reaches the 0 point in the design. Therefore, $f_{a}+e_{3} \rightarrow V_{2}$.

\section{Step 3}

Define $\tilde{\gamma}_{3}=\gamma_{3}-\hat{\gamma}_{3}$ as the estimation error of the upper bound of the external disturbance change, then there is $\dot{\tilde{\gamma}}_{3}=-\dot{\hat{\gamma}}_{3}$. Select the Lyapunov candidate function i.e.

$$
v_{3}=\frac{1}{2} e_{3}^{2}+\frac{1}{2 \delta_{3}} \tilde{\gamma}_{3}^{2}
$$

Deriving equation (19) and substituting equations (13) and (14) we get

$$
\dot{v}_{3} \leq\left(\gamma_{3}-\beta_{3}\right)\left|e_{3}\right|-\frac{1}{\delta_{3}} \tilde{\gamma}_{3} \dot{\hat{\gamma}}_{3}
$$

Assume $\beta_{3}=\hat{\gamma}_{3}+\varepsilon_{3}, \dot{\gamma}_{3}=\delta_{3}\left|e_{3}\right|, \varepsilon_{3}$ is the normal number. By subsituting equation (20), we attain $\dot{v}_{3}=-\varepsilon_{3}\left|e_{2}\right| \leq 0$ representing that $e_{3} \&$ its derivative are in step 2 which reaches the 0 point in the design. Therefore, $\hat{f}_{a} \approx V_{2}$.

\section{Simulation results and analysis}

In this section, the purpose of simulation experiments is to simulate the system over a fixed point. It is proved that the designed sliding mode position controller is better than the tradtional one. It also has a fast convergence speed. The controller can track the desired state with small tracking error and with better anti-interference ability. Table 1 shows the sliding mode controller parameters.

Table 1: The parameter of quadrotor are as follows

$g=9.8 \frac{\mathrm{m}}{\mathrm{s}^{2}}$
$d=0.180 \mathrm{~m}$
$m=2.59 \mathrm{~kg}$
$k_{T}=1.256 e-5 \mathrm{~N} /(\mathrm{rad} / \mathrm{s})^{2}$
$k_{Q}=1.648 e-7 \mathrm{~N} . \mathrm{m} /(\mathrm{rad} / \mathrm{s})^{2}$




$$
\begin{gathered}
J_{x}=1.770 e-2 \mathrm{~kg} \cdot \mathrm{m}^{2} \\
J_{y}=1.77 e-2 \mathrm{~kg} . \mathrm{m}^{2} \\
J_{z}=3.2120 e-2 \mathrm{~kg} . \mathrm{m}^{2} \\
T_{m}=0.156 \mathrm{~s}
\end{gathered}
$$

There are two types of sub models of quadrotor UAV (i) inner loop (ii) outer loop. The first one is for attitude control and the other is for altitude control. When these both models/loops are combined, results in successful flight as per required position and direction. The rotational sub system is used to control and stabilize the initial errors. With the certain oscillations, the switching of input among the steady and transient state set between 0 to $2 \mathrm{sec}$. Figure $3 \mathrm{c}$ shows the hovering scenario with required altitude. The translational and rotational velocity components are shown in the figure 4 and 5 respectively. The further positional axis are equal to 0 . For each simulation, the sampling time was $0.2 \mathrm{sec}$. The maximum time for each simulation is $10 \mathrm{sec}$.

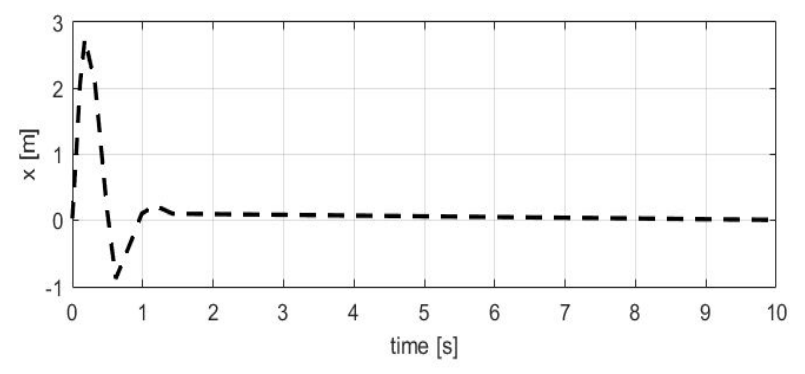

(a)

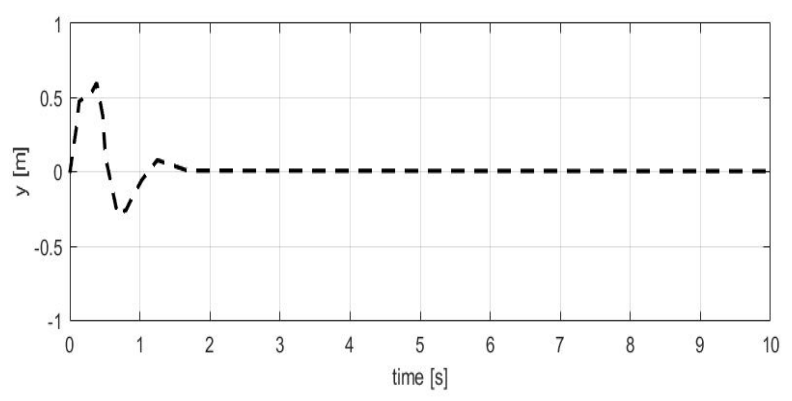

(b)

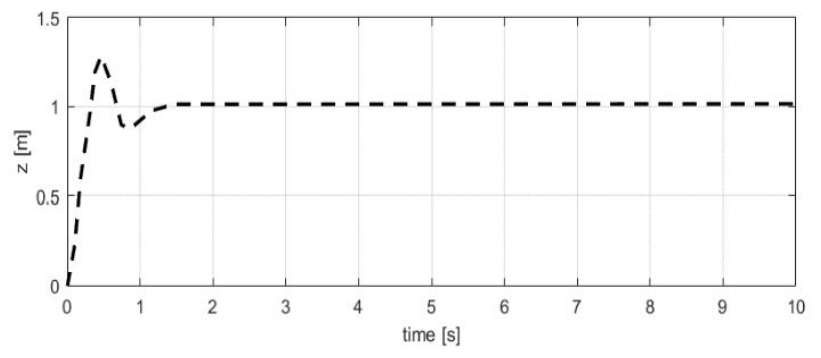

(c) 
Fig. 3 (a, b, c): The position of UAV

Initially, there are many oscillations in the attitude angles as shown in the simulation results. When the desired input command is given, it converges to zero after some seconds. With the help of trim point condition, the altitude of quadrotor UAV is locked at about $1 \mathrm{~m}$. Figure 4 and 5 shows that due to no moment in flights dynamic, the velocity components go to zero.

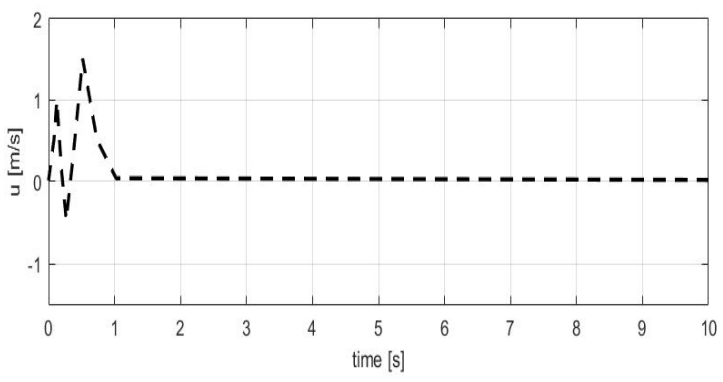

(a)

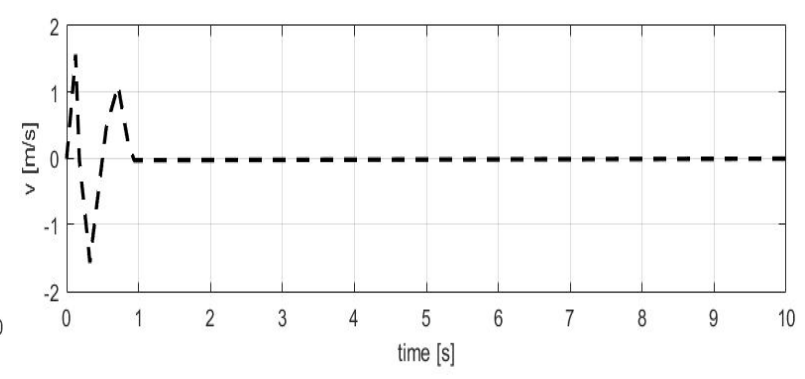

(b)

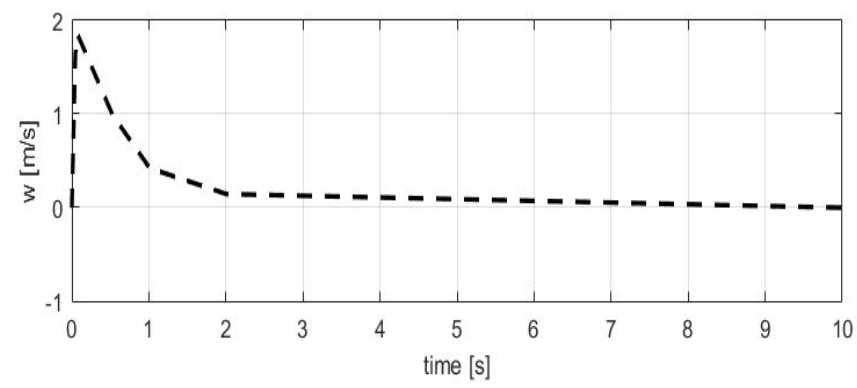

(c)

Fig. 4(a, b, c): The translational velocity components

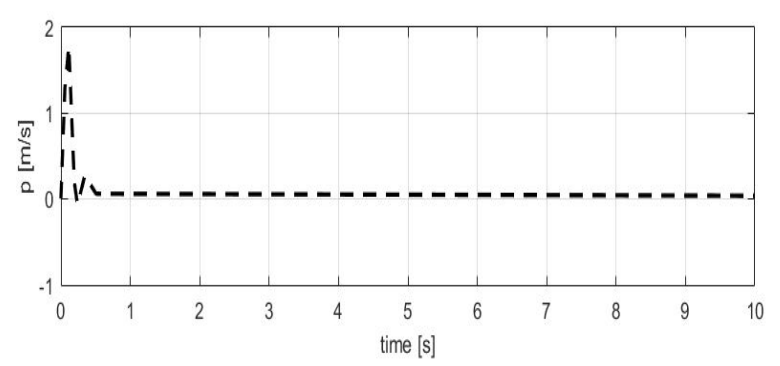

(a)

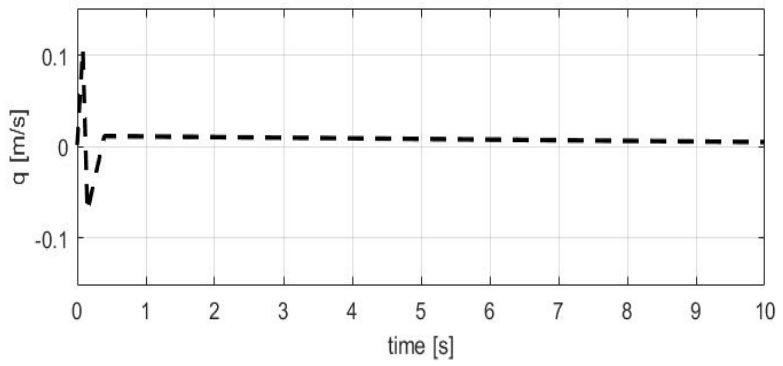

(b) 


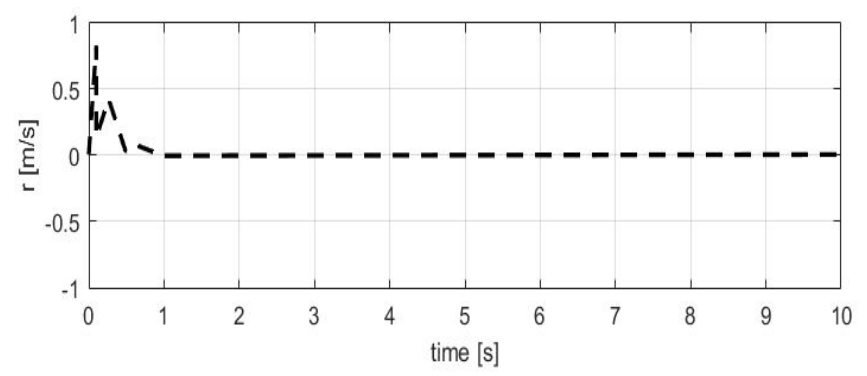

(c)

Figure $5(a, b, c)$ : The rotational velocity components

\section{Conclusion}

In this research article, it is verified that the non-linear behavior of quadrotor UAV is controlled by the designed control algorithm. The required altitude and the hovering state are attain by the inner and outer control loops. The simulation results proved the validity of the controller and shows how efficient the designed algorithm is. It is also observed that the hovering state is achieved with best steady state response. Lastly, the quadrotor quickly converges to the required path in limited time.

\section{References}

1. Wilson Medina-Pazmiño, Aníbal Jara-Olmedo, and David Valencia-Redrován. "Analysis and determination of minimum requirements for a data link communication system for unmanned aerial vehicles- UAV's." 2016.

2. Domingos, Diego, Camargo, Guilherme, and Gomide, Fernando. "Autonomous Fuzzy Control and Navigation of Quadcopters." Ifac Papersonline 49.5:73-78.

3. Scallion, William I, and C. D. J. Cone. "Wind-tunnel Investigation of the Low-speed Stability and Performance Characteristics of a Jet-powered Low-aspect-ratio Vertical-take-off-and-landing Configuration with Engines Buried in Tiltable Wings." (1958).

4. Daniele Palossi, Francesco Conti, and Luca Benini. "An Open Source and Open Hardware Deep Learning-Powered Visual Navigation Engine for Autonomous Nano-UAVs." 2019 15th International Conference on Distributed Computing in Sensor Systems (DCOSS) 2019.

5. Laihong Zhou等. "Quadrotor UAV flight control via a novel saturation integral backstepping controller." Automatika 60.2(2019):193-206.

6. Meng Joo Er, and Ya Lei Sun. "Hybrid fuzzy proportional-integral plus conventional derivative control of linear and nonlinear systems." IEEE Transactions on Industrial Electronics 48.6:1109-1117.

7. Su, Qingyu. "Improved adaptive backstepping sliding mode control for generator steam valves of non-linear power systems." IET Control Theory \& Applications 11.9:1414-1419.

8. Wang, Dao Bo, Zain Anwar Ali, and Muhammad Aamir. "Hybrid Strategy for Nonlinear Control of (6 DOF) Under Actuated Tricolor UAV." International Journal of Control and Automation 9, no. 12 (2016): 463-472.

9. Ya-Fu Peng, Ming-Hung Lin, and Chao-Ming Chong. "Design of Output Recurrent CMAC Backstepping Control System for Tracking Periodic Trajectories." Neural Networks, 2006. IJCNN '06. International Joint Conference on IEEE Xplore, 2006. 


\section{Gyancity Journal of Electronics and Computer Science, \\ Vol.4, No.2, pp. 1-12, September 2019 \\ ISSN: 2446-2918 DOI: 10.21058/gjecs.2019.42005}

10. Chenliang Wang等. "Distributed Adaptive Containment Control for a Class of Nonlinear Multiagent Systems With Input Quantization." IEEE Transactions on Neural Networks \& Learning Systems PP.99(2017):1-10.

11. Suárez, Alejandro M., Duarte-Mermoud, Manuel A., and Bassi, Danilo F. "A predictive control scheme based on neural networks." Kybernetes 35.10:1579-1606.

12. Ali, Zain Anwar, Muhammad Aamir, Dao Bo Wang, and Muhammad Anwar. "Controlling the Dynamics and Stabilization of the Altitude and Attitude of An Underactuated Tri-rotor UAV." International Journal of Control and Automation 10, no. 3 (2017): 303-320.

13. Kordonis, Ioannis, and Papavassilopoulos, George P. "Dynamic games among agents with partial information of the structure of the interactions graph: Decision making and complexity issues." 2013.

14. Derafa, L., Ouldali, A., Madani, T., \& Benallegue, A. . Non-linear control algorithm for the four rotors uav attitude tracking problem. The Aeronautical Journal, 115(1165), 175-185.

15. Ashfaq Ahmad Mian, and Wang Daobo. "Nonlinear Flight Control Strategy for an Underactuated Quadrotor Aerial Robot." Networking, Sensing and Control, 2008. ICNSC 2008. IEEE International Conference on IEEE, 2008.

16. Satoshi Kohno, and Kenji Uchiyama. "Design of robust controller of fixed-wing UAV for transition flight." 2014 International Conference on Unmanned Aircraft Systems (ICUAS) 2014.

17. Askar Azizi, Hamid Nourisola, and Amir Rikhtehgar Ghiasi. "3D inertial algorithm of SLAM for using on UAV." 2016 4th International Conference on Robotics and Mechatronics (ICROM) IEEE, 2016. 